\title{
Smart System for Crowd Monitoring and Detection to Prevent Covid19
}

\author{
Prof. Gaurav Tiwari ${ }^{1}$, Ajay Darakhe², Nikhil Chaudhari², Omkar More ${ }^{2}$ \\ ${ }^{1}$ Professor, Department of Electronics and Telecommunication Engineering, D.Y.P.S.O.E, Pune, Maharashtra, \\ India \\ ${ }^{1}$ Student, Department of Electronics and Telecommunication Engineering, D.Y.P.S.O.E, Pune, Maharashtra, \\ India
}

\section{Article Info}

Volume 8, Issue 3

Page Number: 355-359

Publication Issue :

May-June-2021

\section{Article History}

Accepted : 06 June 2021

Published: 12 June 2021

\section{ABSTRACT}

It is essential to maintain social distance and avoid large mob gatherings at one place to break the chain of corona virus infection, but maintaining these things is not that much easy. People knowingly or unknowingly, gather, roam on the streets \& break the rules. Hence Keeping an eye on all these activities is not an easy job. The proposed system is an automatic method for controlling crowd in this pandemic situation, where crowd gatherings should be avoided on large basis. We have proposed a system which will keep a watchful eye on crowd gathering with help of RPi camera, as crowd is detected the system will give a alert to authorities that they will take actions against the crowd gatherings and restrict the public from areas where crowds are restricted to be gather. The main aim of the survey is to be found how to avoid unnecessary gatherings where crowd is restricted! and if crowd gathers unnecessarily, system can alert the respective authorities and crowd can be minimized.

Keywords: RaspberryPi4, RPi camera module, Python, OpenCV, Haar cascade, Raspbian.

\section{INTRODUCTION}

In December 2019 corona virus first case was found in Wuhan China, around 122 Countries in world are infected. Corona virus infection transfers from human to human and high risk of spread is in public places where people use to gather frequently. COVID-19 spread is getting in control and vaccination is also started but precautions are ignored at most of the places and large amount of crowd gathering are being created by public unnecessarily! Crowd gatherings can be avoided at places like school premises, public transport vehical stops, Government offices etc. which will definitely help in minimising the risk of spreading covid-19 again which is in some of control now! As schools, colleges, government offices , companies are reopening more precautions are needed to be taken. The authorities need reliable 
technology which can survey such places where crowd gathers more and more and to prevent these types of unnecessary movements.

\section{LITERATURE SURVEY}

We have reviewed several papers published by many authors researchers in the international conferences and journals. These papers contain similar concepts and points which are useful for our work. Literature survey is as follow:

[1] this paper describes the machine learning for visual object detection which is able to process images so rapidly and achieving high detection rates, which describes about haar cascade model. It has three main contributions as image representation, learning algorithm based on adaboost and combining complex classifiers in cascade.[2] the paper contains the new human face detection algorithm they have worked on mouth, eye and skin hue by combining the haar cascade classifier and the weak classifiers on the bases of human face.[3] face recognition in real time is so challenging so the this paper proposes principal component analysis for facial recognition system, they developed a camera that is based on real-time face recognition and set an algorithm by developing programming on OpenCV, Haar Cascade, Eigenface, Fisher Face, LBPH, and Python.[4] node classifiers in the cascade detector are been designed in this paper, authors made three contributions as categorization of asymmetries, forward feature selection (FFS) algorithm and a fast precomputing strategy for AdaBoost. FFS, linear asymmetric classifier (LAC). with these contributions the classifiers performance has been improved.[5] gender classification under non uniform illumination variations ha been proposed in this paper it is designed as that it would be fast and low time consuming, Haar Cascade Classifiers are used for face detection from an image.Haar Cascade Classifiers and geometrical properties of facial features are combined for Facial feature extraction from detected face. Support Vector Machine (SVM) is used.[6] using biometric identification for the face recognition is main problem it is lengthy process and the accuracy of the results is not that much clear, Haar Cascades and Eigenface methods are used for fast face recognition, which can detect multiple faces. There system was capable of recognize multiple faces with $91.67 \%$ accuracy level in a single detection process.[7]authors have done the comparison between VJ and HOG for detection of the faces, with that Integral Image through Haar-like feature is calculated by V-J method, by using AdaBoost process to make a robust cascade classifier, authors were motivated by Viola-Jones Haar Cascade Classifier.[8] Haar cascade classifiers (HCC) is used for the architecture of a hierarchical face and eyes detection system, author compared the efficiency of other systems face and eyes HCCs and got their 94\% successful results.[9] Multi-block Local Binary Patterns (MB-LBP), for detecting the face are been presented in this paper, MB-LBP features are used to achieve a goal of detecting face. The authors in this paper mentioned the advantages of MB-LBP feature that boosts the learning for face detection.[10] using the deep transfer learning and the RaspberryPI, authors developed the system and achieved task automation which doesn't need any on ground human effort and reduces man power. accuracy score of $96.02 \%$ is achieved by developers, along with that the authors used the RPi III , Python in this research paper.[11] authors proposed system that will be capable of sensing suspicious events like unknown human face detection, entry in restricted places by capturing videos on basis of the real time. turret mechanism is built using servo motors that will help to camera to rotate in 360' direction, system is automatic as well it can be used manually. Raspberry pi board is used as the central processing unit,[12] In this paper the author has given us the techniques which are been used for crowd monitoring mainly the CCTV crowd flow monitoring is focused by author. Motion-based machine vision techniques for the management of 
large crowds are pointing to control large crowd digitally. Motion based algorithms are mentioned by authors that will detect the crowd.[13] In this paper the Multi-column Convolutional Neural Network (MCNN) architecture is developed by the authors for mapping the image to its crowd density map, this work accurately estimate the crowd count from an individual images, .this work has Raspberry Pi3 board along with that the open $\mathrm{cv}$ and python programming is been used.[14] Covid infections can majorly reduce by maintaining social distancing and less public gathering on these conditions the authors proposed a work where system is able to detect the human pairs, about 6 feet of space between them is assigned for social distancing condition. hybrid combination of a Deep Reinforcement Learning method and traditional model is used to implement this work.[15] circuit television (CCTV) and Drones are used to monitor the crowd on the basis of object detection and compute the distancing between the humans. computer distance algorithms are been used to do this work with that this surveillance method uses Computer vision and Deep learning for croiwd analysis.[16] surveillance robots are proposed in this work which can monitor as well promote the social distancing, trhis system mainly focuses on social distance detection, urban navigation, and intelligent voice interaction. Raspberry $\mathrm{Pi}$, open cv and python based robot system is designed.[17] In this research authors proposed using a monocular camera and deep learning-based real-time object detectors for monitoring the social distancing in public areas, 3 different pedestrian crowd datasets were used to verify the proposed system. This system is real time. conducting social distancing monitoring and finding the critical density was the main concern. Only the pedestrians within the ROI were considered. [18] this system comprises of many of the sensor nodes communicating with a server which is centralized, the work counts number of people entering and leaving a specific zone, checks for physical distancing, monitors body temperature and warns attendees and authorities of violations. They used the Raspberry Pi system to overcome this work with that python programming also used with the crowd detection models.[19] multi-class support vector machine (SVM) running over Raspberry pi (RPi) was used to detecte real-time violence in surveillance videos. six classes like blast, fight, knife, pistol etc. were used along with the SURF feature and it was very effective in real time.[20] Message oriented architecture is used to sense and process modules on bases of real time video captured. Modules like motion detection, background subtraction, people counting and a tracking were used. Author successfully implemented the crowd monitor system using average resolution cameras and the Raspberry Pi control unit.

\section{III.PROPOSED SYSTEM}

After Observing survey we saw that no system is developed such will monitor crowd gatherings and alert the authorities \& monitor the body temperatures fully automatic, that will help to prevent COVID19 indirectly, keeping reference of all above papers we get motivated to do such a work that will find a reliable system.

\section{IV.CONCLUSION}

A Smart system for crowd monitoring and detection to prevent covid19 will effective work for handle the crowded places and monitoring the body temperatures automatically in real time and according to literature survey the hardware and technology which will be used to accomplish this project are Raspberry Pi 4 board as control unit, Rpi camera module, MLX90614 temperature sensor, speakers. Programming will be done in python. This will conclude the overall work which needs to be done to accomplish the result. If we implement this on Broadway, it will very successful in indirectly 
preventing covid19. The main advantage of this smart system is its simplicity and its ability to warn the crowd as well the authorities.

\section{REFERENCES}

[1]. Rapid Object Detection using a Boosted Cascade of Simple Features, paul viola, Michael jeffery jones, FEB2001 Proceedings / CVPR, IEEE Computer Society Conference on Computer Vision and Pattern Recognition. IEEE Computer Society Conference on Computer Vision and Pattern Recognition.

[2]. Human face detection algorithm via Haar cascade classifier combined with three additional classifiers Li Cuimei, Qi Zhiliang, Jia Nan, Wu Jianhua 2017 13th IEEE International Conference on Electronic Measurement \& Instruments (ICEMI), 483-487, 2017

[3]. Face Detection and Recognition Using OpenCV Maliha Khan, Sudeshna Chakraborty, Rani Astya, Shaveta Khepra 2019 International Conference on Computing, Communication, and Intelligent Systems (ICCCIS), 116-119, 2019

[4]. Hybrid approach of haar cascade classifiers and geometrical properties of facial features applied to illumination invariant gender classification system Priyanka Goel, Suneeta Agarwal 2012 International Conference on Computing Sciences, 132-136, 2012

[5]. " Multi-faces recognition process using Haar cascades and eigenface methods Teddy Mantoro, Media A Ayu 018 6th International Conference on Multimedia Computing and Systems (ICMCS), 1-5, 2018

[6]. Comparison of Viola-Jones Haar Cascade classifier and histogram of oriented gradients (HOG) for face detection C Rahmad, RA Asmara, DRH Putra, I Dharma, H Darmono, I Muhiqqin IOP conference series: materials science and engineering 732 (1), 012038, 2020
[7]. The architecture and performance of the face and eyes detection system based on the Haar cascade classifiers Andrzej Kasinski, Adam Schmidt Pattern Analysis and Applications 13 (2), 197-211, 2010

[8]. Face detection based on multi-block lbp representation Lun Zhang, Rufeng Chu, Shiming Xiang, Shengcai Liao, Stan Z Li International conference on biometrics, 11-18, 2007

[9]. Fast asymmetric learning for cascade face detection Jianxin $\mathrm{Wu}, \mathrm{S}$ Charles Brubaker, Matthew D Mullin, James M Rehg IEEE transactions on pattern analysis and machine intelligence 30 (3), 369-382, 2008

[10]. LIVE FACEMASK DETECTION SYSTEM Harsh Nagoriya, Mohak Parekh, researchgate.net 2020.

[11]. Object Detection and Tracking Turret based on Cascade Classifiers and Single Shot Detectors Pritom Gogoi; Manpa Barman; Mahendra Deka; Upasana Rajkonwar; Rhittwikraj Moudgollya, IEEE Published in: 2020 International Conference on Computational Performance Evaluation (ComPE).

[12]. Motion-based machine vision techniques for the management of large crowds B.A. Boghossian; S.A. Velastin, IEEE Published in: ICECS'99. Proceedings of ICECS '99. 6th IEEE International Conference on Electronics, Circuits and Systems (Cat. No.99EX357) Year 2002.

[13]. Single-Image Crowd Counting via MultiColumn Convolutional Neural Network Yingying Zhang, Desen Zhou, Siqin Chen, Shenghua Gao, IEEE Conference on Computer Vision and Pattern Recognition (CVPR), 2016.

[14]. COVID-Robot: Monitoring Social Distancing Constraints in Crowded Scenarios, Adarsh Jagan Sathyamoorthy, Utsav Patel, Yash Ajay Savle, Moumita Paul, Dinesh Manocha, 
Publication:eprint

arXiv:2008.06585Pub

Date:August 2020.

[15]. Monitoring Social Distancing for Covid-19 Using OpenCV and Deep Learning Rucha Visal, Atharva Theurkar, Bhairavi Shukla, International Research Journal of Engineering and Technology (IRJET) Volume: 07 Issue: 06 | June 2020.

[16]. Autonomous Social Distancing in Urban Environments using a Quadruped Robot Tingxiang Fan, Zhiming Chen, Xuan Zhao, Jing Liang, Cong Shen, Dinesh Manocha, Jia Pan, Wei Zhang, arxiv.org august 2020.

[17]. A Vision-based Social Distancing and Critical Density Detection System for COVID-19 Dongfang Yang* Ekim Yurtsever* Vishnu Renganathan Keith A. Redmill Umit “ Ozg * uner, arxiv.org, jul2020.

[18]. Human detector and counter using raspberry Pi microcontroller Shubham Mathur; Balaji Subramanian; Sanyam Jain; Kajal Choudhary; D Rama Prabha, 2017 Innovations in Power and Advanced Computing Technologies (i-PACT).

[19]. Real-time Violence Detection in Surveillance Videos using RPiMuhammad Salman Hikmat Yar, Tauseef Jan, Khalil Ur Rahman, The 5th International Conference on Next Generation Computing 2019.

[20]. Counting and tracking people in a smart room: An IoT approach Dimitris Sgouropoulos; Evaggelos Spyrou; Giorgos Siantikos; Theodoros Giannakopoulos, 2015 10th International Workshop on Semantic and Social Media Adaptation and Personalization (SMAP).

\section{Cite this article as :}

Prof. Gaurav Tiwari, Ajay Darakhe, Nikhil Chaudhari, Omkar More, "Smart System for Crowd Monitoring and Detection to Prevent Covid19", International Journal of Scientific Research in Science, Engineering and Technology (IJSRSET), Online ISSN : 2394-4099, Print ISSN : 2395-1990, Volume 8 Issue 3, pp. 355-359, May-June 2021. Available at doi : https://doi.org/10.32628/IJSRSET218369 Journal URL : https://ijsrset.com/IJSRSET218369 